\title{
¿La percepción del riesgo afecta la forma en que practicamos la oftalmología? A propósito de la pandemia de COVID-19
}

\section{Does risk perception affect the way we practice ophthalmology? About the COVID-19 pandemic}

\author{
Juan P. Olivares-de Emparan* \\ Asociación para Evitar la Ceguera en México, Ciudad de México, México
}

El brote de la enfermedad por coronavirus 2019 (COVID-19), causado por el virus del síndrome respiratorio agudo severo por coronavirus tipo-2 (SARSCoV-2), fue identificado en los primeros días del presente año en Wuhan, China; y declarado pandemia en marzo de $2020^{1}$. Al finalizar este artículo (7 de septiembre de 2020), el número contabilizado de decesos ascendía a 891,308 en el mundo, con 67,781 registrados en México, según cifras oficiales².

En 2003, la pandemia del síndrome respiratorio agudo severo (SARS) afectó de manera importante a profesionales de la salud, representando el $21.1 \%$ de los casos $(1,076 / 8,096)^{3}$. Diferencias en el número de casos reportados en cada país, tasa de pruebas, desempeño de los sistemas de información y el rápido incremento del número de infecciones dificultan conocer con exactitud la prevalencia de SARS-CoV-2 entre el personal de salud ${ }^{4}$. Ing, et al. realizaron una revisión de la literatura acerca de los decesos reportados entre personal médico al 25 de abril, y encontraron un total de 254 muertes, de las cuales, 7 (4\%) correspondían a médicos oftalmólogos 5 . Estos autores no encontraron diferencia en el riesgo de los oftalmólogos al compararse con otras especialidades ${ }^{5}$; sin embargo, la cercanía con el paciente durante las actividades clínicas y quirúrgicas representa un riesgo en el ejercicio de la especialidad 6 .
Adicionalmente, existe la sospecha de la excreción del virus a través de la lágrima o conjuntiva ${ }^{7}$. En una revisión sistemática de la literatura, en la cual se clasificó la información publicada hasta el 21 de abril respecto a COVID-19 y oftalmología, encontramos que los niveles de evidencia para apoyar esta teoría son bajos y aún existen interrogantes del riesgo de transmisión a través de la lágrima o las secreciones conjuntivales. Así mismo, la evidencia relacionada con la frecuencia y características de las manifestaciones oculares del COVID-19 aún es escasa, y se requiere una mayor comprensión para conocer a profundidad los efectos oculares del virus ${ }^{8}$.

Dos publicaciones del presente número de la Revista Mexicana de Oftalmología están dedicados a temas relacionados con COVID-19; las dos están basadas en encuestas que recogen información referente a la percepción de médicos oftalmólogos ante la enfermedad $y$, por lo tanto, a la manera en que como médicos afrontamos la nueva realidad de la práctica de nuestra especialidad. López-Ulloa, et al. realizaron una encuesta del impacto de la pandemia en los oftalmólogos de México. Un total de 241 (66.6\%) participantes mencionaron que los oftalmólogos tenían un mayor riesgo de contagio en comparación con otras especialidades médicas; $103(28.5 \%)$ creían que era similar, y $18(4.9 \%)$, que era menor. Los participantes que tenían

Correspondencia:

*Juan P. Olivares-de Emparan

Vicente García Torres, 46

Col. San Lucas Coyoacán

Fecha de recepción: 09-07-2020

Fecha de aceptación: 10-07-2020

E-mail: olivares.juanpablo@gmail.com

0187-4519/C 2020 Sociedad Mexicana de Oftalmología. Publicado por Permanyer. Este es un (http://creativecommons.org/licenses/by-nc-nd/4.0/).

Disponible en internet: 09-11-2020 Rev Mex Oftalmol. 2020;94(6):245-246 www.rmo.com.mx
ncia CC BY-NC-ND encia CC BY-NC-ND 
más de 30 años de práctica tenían mayor probabilidad de creer que el riesgo era mayor, mientras que los oftalmólogos más jóvenes creían que el riesgo era similar a otras especialidades (J.A López-Ulloa, comunicación personal, 1 julio 2020). La encuesta de MierBolio, et al. recoge datos de la prevalencia de ansiedad en médicos oftalmólogos en un centro de referencia en la ciudad de México utilizando dos escalas diferentes. La prevalencia encontrada fue del $48 \%$ y el $83 \%$, según el instrumento de medición, y la mayoría de los casos fueron clasificados como leves ${ }^{9}$.

La percepción del riesgo es un fenómeno social construido a partir de experiencias previas y es difícil de predecir con certeza. Estudios cualitativos en profesionales de la salud la han relacionado con la disposición de respuesta ante emergencias sanitarias por otros patógenos ${ }^{10,11}$. Gee, et al. realizaron un estudio de entrevistas a profundidad en personal de salud durante el brote de ébola en África en 2014-2016 y mencionan como factores que aumentan la percepción del riesgo: la impresión general de la sociedad y la familia, la información en medios de comunicación y las infecciones en colegas. Por otro lado, el conocimiento y la confianza en las instituciones mitigan dicha percepción ${ }^{9}$. Balicer, et al. encontraron que hasta la mitad del personal de salud podría ausentarse ante una crisis sanitaria por influenza en Maryland, EE.UU. ${ }^{11}$.

El impacto que genera la percepción del riesgo en los profesionales de salud y su respuesta ante las situaciones de crisis son factores que repercuten en el sistema de salud y, por lo tanto, en la carga de enfermedades en la sociedad. Las políticas de control en nuestras instituciones deberán encaminarse a incrementar la autodeterminación, el conocimiento y la certidumbre entre el personal de salud a fin de que dicha percepción resulte en el menor perjuicio posible en la salud visual de nuestra población.

\section{Bibliografía}

1. Wu D, Wu T, Liu Q, Yang, Z The SARS-CoV-2 outbreak: what we know. Int J Infect Dis. 2020;94:44-8.

2. Department of Civil and Systems Engineering - Johns Hopkins University. COVID-19 Dashboard. COVID-19 Dashboard by the CSSE at Johns Hopkins University. 2020. Disponible en: https://gisanddata.maps.arcgis. com/apps/opsdashboard/index.html\#/bda $7594740 \mathrm{fd}$ 40299423467b48e9ecf6.

3. World Health Organization. Summary of probable SARS cases with onset of illness from 1 November 2002 to 31 July 2003. WHO; 2004. Disponible en: https://www.who.int/csr/sars/country/table2004_04_21/en/

4. Lahner E, Dilaghi E, Prestigiacomo C, Alessio G, Marcellini L, Simmaco M, et al. Prevalence of Sars-Cov-2 Infection in Health Workers (HWs) and Diagnostic Test Performance : The Experience of a Teaching Hospital in Central Italy. Int J Environ Res Public Health. 2020;17(12):4417.

5. Ing EB, Xu QA, Salimi A, Torun N. Physician death from coronavirus (COVID-19) disease. Occupational Medicine. 2020;70(5):370-4

6. American Academy of Ophthalmology. Alert : Important coronavirus updates for ophthalmologists. 2020. Disponible en: https://www.aao.org/ headline/alert-important-coronavirus-context.

7. Lu CW, Liu XF, Jia ZF. 2019-nCoV transmission through the ocular surface must not be ignored. Lancet. 2020;395:e39.

8. Olivares-de Emparan JP, Sardi-Correa C, López-Ulloa JA, Viteri-Soria J, Penniecook JA, Jimenez-Román J, et al. COVID-19 and the eye: how much do we really know? A best evidence review. Arq Bras Oftalmol. 2020;83:250-61

9. Mier-Bolio JR, Arroyo-González JM, Baques-Guillen E, et al. COVID-19 y ansiedad en oftalmólogos. Rev Mex Oftalmol. 2020;94(6): XXXXXX

10. Gee $S$, Skovdal M. The role of risk perception in willingness to respond to the 2014-2016 West African Ebola outbreak: a qualitative study of international health care workers. Glob Health Res Policy. 2017:2:1-10.

11. Balicer RD, Omer SB, Barnett DJ, Everly GS. Local public health workers' perceptions toward responding to an influenza pandemic. BMC Public Health. 2006;6:1-8. 